\title{
Customization of software as a service application: problems and objectives
}

\begin{abstract}
The key feature of SaaS application is that it addresses the needs of many different customers using only one software, rather than multiple developments and versions, a concept known as multi-tenancy. However, it typically results in a one-size-fits-all approach and the application only fulfills the requirements that are generic to all tenants. Therefore, a multi-tenancy SaaS application is reliant upon an ability to be customized in order to be successful. Responding to distinctive needs of each customer and maintaining the key feature of developing SaaS application creates complexity in SaaS customization. This paper delves into some challenges related to SaaS customization, and then maps among these challenges in order to structure the problem and objective trees of SaaS customization. This paper may serve as a step towards reducing complexity in SaaS customization.
\end{abstract}

Keyword: Software as a service; Customization; Multitenant; Problem tree; Objective tree 